\title{
DIE EXPLOSIVSTOFFE
}

MIT BESONDERER BERÜCKSICHTIGUNG

DER NEUEREN PATENTE

ERSTES HEFT

\section{SCHWARZPULVER UND SPRENGSALPETER}

VON

\section{DR. RICHARD ESCALES}

MIT ZAHLREICHEN ABBILDUNGEN UND EINER TAFEL

ZWEITE, VÖLLIG UMGEARBEITETE UND ERWEITERTE AUfLAGE

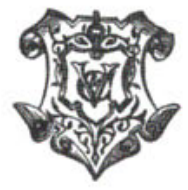

LEIPZIG

VERLAG VON VEIT \& COMP.

1914 


\title{
DIE EXPLOSIVSTOFFE
}

MIT BESONDERER BERÜCKSICHTIGUNG

DER NEUEREN PATENTE

\author{
BEARBEITET \\ voN \\ DR. RICHARD ESCALES
}

ERSTES HEFT
SCHWARZPULVER UND SPRENGSALPETER

ZWEITE, VÖLLIG UMGEARBEITETE UND ERWEITERTE AUFLAGE

LEIPZIG

VERLAG VON VEIT \& COMP.

1914 


\section{SCHWARZPULVER UND SPRENGSALPETER}

VON

DR. RICHARD ESCALES

MIT ZAHLREICHEN ABBILDUNGEN UND EINER TAFEL

ZWEITE, VöLLIG UMGEARBEITETE UND ERWEITERTE AUfLAGE

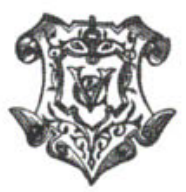

LEIPZIG

VERLAG VON VEIT \& COMP.

1914 
